\title{
Pacific
}

Journal of

Mathematics

\section{SURFACES IN THE 3-DIMENSIONAL LORENTZ-MINKOWSKI} SPACE SATISFYING $\Delta x=A x+B$

\author{
Luis Alías, Angel Ferrandez and Pascual LuCAS
}




\section{SURFACES IN THE 3-DIMENSIONAL LORENTZ-MINKOWSKI SPACE SATISFYING$$
\Delta x=A x+B
$$

\section{Luis J. Alías, Angel Ferrández and Pascual lucas}

In this paper we locally classify the surfaces $M_{s}^{2}$ in the 3-dimensional Lorentz-Minkowski space $\mathbb{L}^{3}$ verifying the equation $\Delta x=$ $A x+B$, where $A$ is an endomorphism of $\mathbb{L}^{3}$ and $B$ is a constant vector.

We obtain that classification by proving that $M_{s}^{2}$ has constant mean curvature and in a second step we deduce $M_{s}^{2}$ is isoparametric.

0. Introduction. In [FL90] the last two authors obtain a classification of surfaces $M_{s}^{2}$ in the 3-dimensional Lorentz-Minkowski space satisfying the condition $\Delta H=\lambda H$, for a real constant $\lambda$, where $H$ is the mean curvature vector field. That equation is nothing but a system of partial differential equations, so that the problems quoted in [FL90] can be framed in a more general situation: classify semi-Riemannian submanifolds by means of some characteristic differential equations. In this line, the technique of finite type submanifolds, created and developed by B. Y. Chen, has been shown as a fruitful tool to inquire into not only the intrinsic configuration of the submanifold, but also the extrinsic one, because the Laplacian of the isometric immersion is essentially the mean curvature vector field of the submanifold.

Following Chen's idea, Garay [Gar88] has obtained a characterization of connected, complete surfaces of revolution in $\mathbb{E}^{3}$ whose component functions in $\mathbb{E}^{3}$ are eigenfunctions of its Laplacian with possibly distinct eigenvalues. In a second step, in [Gar90], Garay found that the only Euclidean hypersurfaces whose coordinate functions are eigenfunctions for its Laplacian are open pieces of a minimal hypersurface, a hypersphere or a generalized circular cylinder.

More recently, in [DPV90], Dillen-Pas-Verstraelen pointed out that Garay's condition is not coordinate invariant as a circular cylinder in $\mathbb{E}^{3}$ shows. Then they study and classify the surfaces in $\mathbb{E}^{3}$ which satisfy $\Delta x=A x+B$, where $\Delta$ is the Laplacian on the surface, $x$ represents the isometric immersion in $\mathbb{E}^{3}, A \in \mathbb{E}^{3 \times 3}$ and $B \in \mathbb{R}^{3}$.

It is well known that when the ambient space is the 3-dimensional 
Lorentz-Minkowski space $\mathbb{L}^{3}$, then the surface $M_{s}^{2}$ can be endowed with a Riemannian metric (spacelike surface) or a Lorentzian metric (Lorentzian surface) and therefore, as we pointed out in [FL90], a richer classification is hoped. So, the following geometric question seems to be coming up in a natural way:

"Which are the surfaces in $\mathbb{L}^{3}$ satisfying the condition $\Delta x=A x+B$, where $A$ is an endomorphism of $\mathbb{L}^{3}$ and $B$ is a constant vector?"

To solve this question we follow the same way of reasoning as in [FL90], which is quite different than that used by Dillen-PasVerstraelen in [DPV90]. We would like to remark that our proof also works in the Riemannian case, so that the Theorem in [DPV90] can be obtained as a consequence of our main result.

1. Some examples. Let $f: \mathbb{L}^{3} \rightarrow \mathbb{R}$ be a real function defined by

$$
f(x, y, z)=-\delta_{1} x^{2}+y^{2}+\delta_{2} z^{2},
$$

where $\delta_{1}$ and $\delta_{2}$ belong to the set $\{0,1\}$ and they do not vanish simultaneously. Taking $r>0$ and $\varepsilon= \pm 1$, the set $f^{-1}\left(\varepsilon r^{2}\right)$ is a surface in $\mathbb{L}^{3}$ provided that $\left(\delta_{1}, \delta_{2}, \varepsilon\right) \neq(0,1,-1)$.

A straightforward computation shows that the unit normal vector field is written as $N=(1 / r)\left(\delta_{1} x, y, \delta_{2} z\right)$ and the principal curvatures are

$$
\mu_{1}=-\delta_{1} / r \quad \text { and } \quad \mu_{2}=-\delta_{2} / r
$$

Then the mean curvature is given by

$$
\alpha=(\varepsilon / 2)\left(\mu_{1}+\mu_{2}\right)=(-\varepsilon / 2 r)\left(\delta_{1}+\delta_{2}\right)
$$

and by using the well-known formula $\Delta x=-2 H=-2 \alpha N$ we obtain $\Delta x=A x$, where

$$
A=\frac{\varepsilon\left(\delta_{1}+\delta_{2}\right)}{r^{2}}\left(\begin{array}{ccc}
\delta_{1} & 0 & 0 \\
0 & 1 & 0 \\
0 & 0 & \delta_{2}
\end{array}\right)
$$

The adjoint table collects all the above possibilities. 
TABLE 1

\begin{tabular}{|c|c|c|}
\hline Equation & Surface & $A$ \\
\hline$y^{2}+z^{2}=r^{2}$ & $\mathbb{L} \times S^{1}(r)$ & $\left(\begin{array}{ccc}0 & 0 & 0 \\
0 & 1 / r^{2} & 0 \\
0 & 0 & 1 / r^{2}\end{array}\right)$ \\
\hline$-x^{2}+y^{2}=-r^{2}$ & $H^{1}(r) \times \mathbb{R}$ & $\left(\begin{array}{ccc}-1 / r^{2} & 0 & 0 \\
0 & -1 / r^{2} & 0 \\
0 & 0 & 0\end{array}\right)$ \\
\hline$-x^{2}+y^{2}=r^{2}$ & $S_{1}^{1}(r) \times \mathbb{R}$ & $\left(\begin{array}{ccc}1 / r^{2} & 0 & 0 \\
0 & 1 / r^{2} & 0 \\
0 & 0 & 0\end{array}\right)$ \\
\hline$-x^{2}+y^{2}+z^{2}=-r^{2}$ & $H^{2}(r)$ & $\left(\begin{array}{ccc}-2 / r^{2} & 0 & 0 \\
0 & -2 / r^{2} & 0 \\
0 & 0 & -2 / r^{2}\end{array}\right)$ \\
\hline$-x^{2}+y^{2}+z^{2}=r^{2}$ & $S_{1}^{2}(r)$ & $\left(\begin{array}{ccc}2 / r^{2} & 0 & 0 \\
0 & 2 / r^{2} & 0 \\
0 & 0 & 2 / r^{2}\end{array}\right)$ \\
\hline
\end{tabular}

2. Setup. Let $M_{s}^{2}$ be a surface in $\mathbb{L}^{3}$ with index $s=0,1$. Throughout this paper we will denote by $\sigma, S, H, \nabla$ and $\bar{\nabla}$ the second fundamental form, the shape operator, the mean curvature vector field, the Levi-Civita connection on $M_{s}^{2}$ and the usual flat connection on $\mathbb{L}^{3}$, respectively. Let $N$ be a unit vector field normal to $M_{s}^{2}$ and let $\alpha$ be the mean curvature with respect to $N$, i.e., $H=\alpha N$.

Let $x: M_{s}^{2} \rightarrow \mathbb{L}^{3}$ be an isometric immersion satisfying the equation

$$
\Delta x=A x+B,
$$

where $A$ is an endomorphism of $\mathbb{L}^{3}$ and $B$ is a constant vector in $\mathbb{L}^{3}$. If we take a covariant derivative in (2.1) and use the well-known equation $\Delta x=-2 H$, by applying the Weingarten formula we have

$$
A X=2 \alpha S X-2 X(\alpha) N,
$$

for any vector field $X$ tangent to $M_{s}^{2}$. From here and the selfadjointness of $S$ one easily gets

$$
\langle A X, Y\rangle=\langle X, A Y\rangle,
$$

for any tangent vector fields $X$ and $Y$.

The covariant derivative in (2.3) yields

$$
\begin{aligned}
& \langle A \sigma(X, Z), Y\rangle-\langle A \sigma(Y, Z), X\rangle \\
& \quad=\langle\sigma(X, Z), A Y\rangle-\langle\sigma(Y, Z), A X\rangle .
\end{aligned}
$$


Now, by applying the Laplacian on both sides of (2.1) and taking into account the formula for $\Delta H$ obtained in [FL90], we have

$$
A H=2 S(\nabla \alpha)+2 \varepsilon \alpha \nabla \alpha+\left\{\Delta \alpha+\varepsilon \alpha|S|^{2}\right\} N,
$$

where $\nabla \alpha$ stands for the gradient of $\alpha$ and $\varepsilon=\langle N, N\rangle$.

As for the structure equations we would like to set the notation that will be used later on. Let $\left\{E_{1}, E_{2}, E_{3}\right\}$ be a local orthonormal frame and let $\left\{\omega^{1}, \omega^{2}, \omega^{3}\right\}$ and $\left\{\omega_{i}^{j}\right\}_{i, j}$ be the dual frame and the connection forms, respectively, given by

$$
\omega^{i}(X)=\left\langle X, E_{i}\right\rangle, \quad \omega_{i}^{j}(X)=\left\langle\bar{\nabla}_{X} E_{i}, E_{j}\right\rangle .
$$

Then we have

$$
d \omega^{i}=-\sum_{j=1}^{3} \varepsilon_{j} \omega_{j}^{i} \wedge \omega^{j}, \quad d \omega_{i}^{j}=-\sum_{k=1}^{3} \varepsilon_{k} \omega_{k}^{j} \wedge \omega_{i}^{k} .
$$

3. The characterization theorem. All exhibited examples in $\S 1$ have constant mean curvature. It seems reasonable to ask for surfaces in $\mathbb{L}^{3}$ satisfying (2.1) having non constant mean curvature. The answer is negative as the following proposition shows.

Proposition 3.1. Let $x: M_{s}^{2} \rightarrow \mathbb{L}^{3}$ be an isometric immersion satisfying $\Delta x=A x+B$. Then $M_{s}^{2}$ has constant mean curvature.

Proof. Let us start with the open set $\mathscr{U}=\left\{p \in M_{s}^{2}: \nabla \alpha^{2}(p) \neq 0\right\}$. We are going to show that $\mathscr{U}$ is empty. Otherwise, we have

$$
\sigma(X, Y)=\varepsilon \frac{\langle S X, Y\rangle}{\alpha} H,
$$

for any tangent vector fields on $\mathscr{U}$. Then from (2.5) we obtain

$$
\langle A \sigma(X, Y), Z\rangle=2 \frac{\langle S X, Y\rangle}{\alpha}(\varepsilon S Z(\alpha)+\alpha Z(\alpha)) \text {. }
$$

Now, by applying (2.2), (2.4) and (3.6) we get

$$
T X(\alpha) S Y=T Y(\alpha) S X,
$$

where $T$ is the self-adjoint operator given by $T X=2 \alpha X+\varepsilon S X$.

Case 1. $T(\nabla \alpha) \neq 0$ on $\mathscr{U}$. Then there exists a tangent vector field $X$ such that $T X(\alpha) \neq 0$, which implies by using (3.7) that $S$ has rank one on $\mathscr{U}$. Thus we can choose a local orthonormal frame $\left\{E_{1}, E_{2}, E_{3}\right\}$ with $S E_{1}=2 \varepsilon \alpha E_{1}, S E_{2}=0$ and $E_{3}=N$. From here and again from (3.7) we have $E_{2}(\alpha)=0$. Let $\left\{\omega^{1}, \omega^{2}, \omega^{3}\right\}$ and $\left\{\omega_{i}^{j}\right\}_{i, j}$ be the dual frame and the connection forms, respectively. It is easy to see that 


$$
\begin{gathered}
\omega_{3}^{1}=-2 \varepsilon \alpha \omega^{1}, \\
\omega_{3}^{2}=0, \\
d \alpha=\varepsilon_{1} E_{1}(\alpha) \omega^{1} .
\end{gathered}
$$

Taking exterior differentiation in (3.8) and using (3.10) and the structure equations we obtain $d \omega^{1}=0$ and therefore we locally have $\omega^{1}=$ $d u$, for a certain function $u$. Now, from (3.10) we get $d \alpha \wedge d u=0$ and then $\alpha$ depends on $u, \alpha=\alpha(u)$, and therefore $E_{1}(\alpha)=\varepsilon_{1} \alpha^{\prime}(u)$.

Taking into account (3.9) and $d \omega^{1}=0$ we deduce $\omega_{2}^{1}=0$. Then we have

$$
\text { (3.11) } \Delta \alpha=-\sum_{i} \varepsilon_{i}\left\{E_{i} E_{i}(\alpha)-\nabla_{E_{i}} E_{i}(\alpha)\right\}=-\varepsilon_{1} E_{1} E_{1}(\alpha)=-\varepsilon_{1} \alpha^{\prime \prime} \text {. }
$$

On the other hand, from (2.2), (2.5) and (3.11) the associated matrix to the endomorphism $A$ with respect to $\left\{E_{1}, E_{2}, N\right\}$ is given by

$$
\left(\begin{array}{ccc}
4 \varepsilon \alpha^{2} & 0 & 6 \varepsilon \alpha^{\prime} \\
0 & 0 & 0 \\
-2 \varepsilon_{1} \alpha^{\prime} & 0 & -\varepsilon_{1} \frac{\alpha^{\prime \prime}}{\alpha}+4 \varepsilon \alpha^{2}
\end{array}\right) .
$$

By considering the invariant elements of $A$, we obtain the following differential equations:

$$
\begin{gathered}
\varepsilon_{1} \alpha^{\prime \prime}=8 \varepsilon \alpha^{3}-\lambda_{1} \alpha, \\
-4 \varepsilon \varepsilon_{1} \alpha \alpha^{\prime \prime}+16 \alpha^{4}+12 \varepsilon \varepsilon_{1}\left(\alpha^{\prime}\right)^{2}=\lambda_{2},
\end{gathered}
$$

where $\lambda_{1}$ and $\lambda_{2}$ are two real constants.

Let us take $\beta=\left(\alpha^{\prime}\right)^{2}$. Then $d \beta / d \alpha=2 \alpha^{\prime \prime}$ and from (3.12) we have

$$
\beta=4 \varepsilon \varepsilon_{1} \alpha^{4}-\lambda_{1} \varepsilon_{1} \alpha^{2}+C,
$$

where $C$ is a constant.

Now, from (3.12) and (3.13) we get

$$
12 \beta=\lambda_{2} \varepsilon \varepsilon_{1}+16 \varepsilon \varepsilon_{1} \alpha^{4}-4 \lambda_{1} \varepsilon_{1} \alpha^{2} .
$$

Finally, we deduce from (3.14) and (3.15) that $\alpha$ is locally constant on $\mathscr{U}$, which is a contradiction.

Case 2. There exists a point $p$ in $\mathscr{U}$ such that $T(\nabla \alpha)(p)=0$. Thus from (2.2) and (2.5) we have

$$
\langle A H, X\rangle(p)=-2 \varepsilon \alpha(p) X(\alpha)(p)=\langle H, A X\rangle(p),
$$

which implies, jointly with (2.3), that $A$ is a self-adjoint endomorphism in $\mathbb{L}^{3}$. Then the above equation remains valid everywhere on 
$\mathscr{U}$ and therefore we get

$$
S(\nabla \alpha)=-2 \varepsilon \alpha \nabla \alpha .
$$

Since $-2 \varepsilon \alpha$ is an eigenvalue of $S$ and $\operatorname{tr} S=2 \varepsilon \alpha$ then $S$ is diagonalizable and we can choose a local orthonormal frame $\left\{E_{1}, E_{2}, E_{3}\right\}$ such that $E_{3}=N, S E_{1}=-2 \varepsilon \alpha E_{1}$ with $E_{1}$ parallel to $\nabla \alpha$ and $S E_{2}=4 \varepsilon \alpha E_{2}$. Let $\left\{\omega^{1}, \omega^{2}, \omega^{3}\right\}$ and $\left\{\omega_{i}^{j}\right\}_{i, j}$ be the dual frame and the connection forms, respectively. Then

$$
\begin{gathered}
\omega_{3}^{1}=2 \varepsilon \alpha \omega^{1}, \\
\omega_{3}^{2}=-4 \varepsilon \alpha \omega^{2}, \\
d \alpha=\varepsilon_{1} E_{1}(\alpha) \omega^{1} .
\end{gathered}
$$

Taking again exterior differentiation in (3.17) and using the structure equations we have $d \omega^{1}=0$. Therefore one locally has $\omega^{1}=d u$, for some function $u$, and thus $\alpha$ depends on $u, \alpha=\alpha(u)$ and $E_{1}(\alpha)=\varepsilon_{1} \alpha^{\prime}$.

By exterior differentiation in (3.18) and using again the structure equations we obtain

$$
3 \varepsilon_{1} \alpha \omega_{2}^{1}=2 \alpha^{\prime} \omega^{2} .
$$

A straightforward computation from (3.20) leads to

$$
3 \alpha \alpha^{\prime \prime}=5\left(\alpha^{\prime}\right)^{2}-36 \varepsilon \varepsilon_{1} \alpha^{4} .
$$

If we put $\beta=\left(\alpha^{\prime}\right)^{2}$ then the last equation can be rewritten as

$$
\frac{3}{2} \alpha \frac{d \beta}{d \alpha}=5 \beta-36 \varepsilon \varepsilon_{1} \alpha^{4}
$$

whose solution is given by

$$
\beta=C \alpha^{10 / 3}-36 \varepsilon \varepsilon_{1} \alpha^{4},
$$

where $C$ is a constant.

On the other hand, from the definition of $\Delta \alpha$, the fact that $E_{1}$ is parallel to $\nabla \alpha$ and (3.20) we obtain

$$
\alpha \Delta \alpha=-\varepsilon_{1} \alpha \alpha^{\prime \prime}+\frac{2 \varepsilon_{1}}{3}\left(\alpha^{\prime}\right)^{2} .
$$

Now, from (2.2) and (2.5) it is easy to get

$$
\alpha \Delta \alpha=\lambda \alpha^{2}-24 \varepsilon \alpha^{4}, \quad \lambda=\operatorname{tr}(A), w
$$


that jointly with (3.24) yields

$$
3 \alpha \alpha^{\prime \prime}=72 \varepsilon \varepsilon_{1} \alpha^{4}-3 \lambda \varepsilon_{1} \alpha^{2}+2\left(\alpha^{\prime}\right)^{2} .
$$

Finally, a similar reasoning as in Case 1 by using now (3.21), (3.23) and (3.25) leads to $\alpha$ is locally constant on $\mathscr{U}$, which is again a contradiction with the definition of $\mathscr{U}$.

Anyway, we deduce $\mathscr{U}$ is empty and then $M_{s}^{2}$ has constant mean curvature.

Now, we are ready to show the main theorem of this paper.

THEOREM 3.2. Let $x: M_{s}^{2} \rightarrow \mathbb{L}^{3}$ be an isometric immersion. Then $\Delta x=A x+B$ if and only if one of the following statements holds true:

(1) $M_{s}^{2}$ has zero mean curvature everywhere.

(2) $M_{s}^{2}$ is an open piece of one of the following surfaces: $\mathbb{L} \times S^{1}(r)$, $H^{1}(r) \times \mathbb{R}, S_{1}^{1}(r) \times \mathbb{R}, H^{2}(r), S_{1}^{2}(r)$.

Proof. Let $M_{s}^{2}$ be a surface in $\mathbb{L}^{3}$ such that $\Delta x=A x+B$. From Proposition 3.1 we know $M_{s}^{2}$ has constant mean curvature $\alpha$. If $\alpha=0$ there is nothing to prove. So, suppose $\alpha \neq 0$. Then from (2.2) and (2.5) we get

$$
\left\{\begin{array}{l}
A X=2 \alpha S X \\
A N=\varepsilon|S|^{2} N
\end{array}\right.
$$

and therefore

$$
\operatorname{tr}(A)=2 \alpha \operatorname{tr}(S)+\varepsilon|S|^{2}=4 \varepsilon \alpha^{2}+\varepsilon|S|^{2},
$$

from which we deduce $|S|^{2}$ is constant and then $M_{s}^{2}$ is an isoparametric surface. If $s=0, M$ is an open piece of $H^{2}(r)$ or $H^{1}(r) \times \mathbb{R}$. When $s=1$, it follows from [Mag85] that $M$ is an open piece of one of the following surfaces: $S_{1}^{2}(r), S_{1}^{1}(r) \times \mathbb{R}, \mathbb{L} \times S^{1}(r)$ and a $B$-scroll. However a straightforward calculation shows that the $B$-scroll does not satisfy the condition $\Delta x=A x+B$.

As we have pointed out in the Introduction, our proof also works when the ambient space is $\mathbb{E}^{3}$. Then the Theorem of Dillen-PasVerstraelen in [DPV90] can be viewed as a consequence of our Theorem:

Corollary 3.3. Let $x: M^{2} \rightarrow \mathbb{E}^{3}$ be an isometric immersion. Then $M$ satisfies $\Delta x=A x+B$ if and only if it is an open piece of a minimal surface, a sphere or a circular cylinder. 


\section{REFERENCES}

[DPV90] F. Dillen, J. Pas and L. Verstraelen, On surfaces of finite type in Euclidean 3-space, Kodai Math. J., 13 (1990), 10-21.

[FL90] A. Ferrández and P. Lucas, On surfaces in the 3-dimensional LorentzMinkowski space, 1990. To appear in Pacific J. Math.

[Gar88] O. J. Garay, On a certain class of finite type surfaces of revolution, Kodai Math. J., 11 (1988), 25-31.

[Gar90] __, An extension of Takahashi's theorem, Geometriae Dedicata, 34 (1990), 105-112.

[Mag85] M. A. Magid, Lorentzian isoparametric hypersurfaces, Pacific J. Math., 118 (1985), 165-197.

Received April 30, 1991. The first author was supported by an FPI Grant, DGICYT, 1990. The second and third authors were partially supported by a DGICYT Grant No. PS 87-0115-C03-03.

Departamento de Matemáticas

UNIVERSIDAD DE MURCIA

CAMPUS DE ESPINARDO

30100 Espinardo, Murcia, Spain 


\title{
PACIFIC JOURNAL OF MATHEMATICS \\ Founded by \\ E. F. BeCKenbach (1906-1982) F. Wolf (1904-1989)
}

\section{EDITORS}

\author{
V. S. VARADARAJAN \\ (Managing Editor) \\ University of California \\ Los Angeles, CA 90024-1555 \\ vsv@math.ucla.edu \\ Herbert Clemens \\ University of Utah \\ Salt Lake City, UT 84112 \\ clemens@math.utah.edu \\ F. Michael Christ \\ University of California \\ Los Angeles, CA 90024-1555 \\ christ@math.ucla.edu \\ THOMAS ENRIGHT \\ University of California, San Diego \\ La Jolla, CA 92093 \\ tenright@ucsd.edu
}

\author{
Nicholas ERcolani \\ University of Arizona \\ Tucson, AZ 85721 \\ ercolani@math.arizona.edu \\ R. FINN \\ Stanford University \\ Stanford, CA 94305 \\ finn@gauss.stanford.edu \\ VAughan F. R. Jones \\ University of California \\ Berkeley, CA 94720 \\ vfr@math.berkeley.edu \\ STEVEN KeRCKHOFF \\ Stanford University \\ Stanford, CA 94305 \\ spk@gauss.stanford.edu
}

\author{
C. C. MOORE \\ University of California \\ Berkeley, CA 94720
}

MARTIN SCHARLEMANN

University of California

Santa Barbara, CA 93106

mgscharl@henri.ucsb.edu

\author{
HAROLD STARK \\ University of California, San Diego \\ La Jolla, CA 92093
}

\section{SUPPORTING INSTITUTIONS}

UNIVERSITY OF ARIZONA
UNIVERSITY OF BRITISH COLUMBIA
CALIFORNIA INSTITUTE OF TECHNOLOGY
UNIVERSITY OF CALIFORNIA
MONTANA STATE UNIVERSITY
UNIVERSITY OF NEVADA, RENO
NEW MEXICO STATE UNIVERSITY
OREGON STATE UNIVERSITY

\author{
UNIVERSITY OF OREGON \\ UNIVERSITY OF SOUTHERN CALIFORNIA \\ STANFORD UNIVERSITY \\ UNIVERSITY OF HAWAII \\ UNIVERSITY OF TOKYO \\ UNIVERSITY OF UTAH \\ WASHINGTON STATE UNIVERSITY \\ UNIVERSITY OF WASHINGTON
}

The Supporting Institutions listed above contribute to the cost of publication of this Journal, but they are not owners or publishers and have no responsibility for its content or policies.

\begin{abstract}
Mathematical papers intended for publication in the Pacific Journal of Mathematics should be in typed form or offset-reproduced (not dittoed), double spaced with large margins. Please do not use built up fractions in the text of the manuscript. However, you may use them in the displayed equations. Underline Greek letters in red, German in green, and script in blue. The first paragraph must be capable of being used separately as a synopsis of the entire paper. In particular it should contain no bibliographic references. Please propose a heading for the odd numbered pages of less than 35 characters. Manuscripts, in triplicate, may be sent to any one of the editors. Please classify according to the 1991 Mathematics Subject Classification scheme which can be found in the December index volumes of Mathematical Reviews. Supply name and address of author to whom proofs should be sent. All other communications should be addressed to the managing editor, or Elaine Barth, University of California, Los Angeles, California 90024-1555.

There are page-charges associated with articles appearing in the Pacific Journal of Mathematics. These charges are expected to be paid by the author's University, Government Agency or Company. If the author or authors do not have access to such Institutional support these charges are waived. Single authors will receive 50 free reprints; joint authors will receive a total of 100 free reprints. Additional copies may be obtained at cost in multiples of 50 .
\end{abstract}

The Pacific Journal of Mathematics (ISSN 0030-8730) is published monthly except for July and August. Regular subscription rate: $\$ 190.00$ a year (10 issues). Special rate: $\$ 95.00$ a year to individual members of supporting institutions.

Subscriptions, orders for numbers issued in the last three calendar years, and changes of address should be sent to Pacific Journal of Mathematics, P.O. Box 969, Carmel Valley, CA 93924, U.S.A. Old back numbers obtainable from Kraus Periodicals Co., Route 100, Millwood, NY 10546.

The Pacific Journal of Mathematics at P.O. Box 969, Carmel Valley, CA 93924 (ISSN 0030-8730) is published monthly except for July and August. Second-class postage paid at Carmel Valley, California 93924, and additional mailing offices. Postmaster: send address changes to Pacific Journal of Mathematics, P.O. Box 969, Carmel Valley, CA 93924.

\section{PUBLISHED BY PACIFIC JOURNAL OF MATHEMATICS, A NON-PROFIT CORPORATION} Copyright (C) 1992 by Pacific Journal of Mathematics 


\section{PACIFIC JOURNAL OF MATHEMATICS}

Volume $156 \quad$ No. $2 \quad$ December 1992

Surfaces in the 3-dimensional Lorentz-Minkowski space satisfying 201 $\Delta x=A x+B$

Luis Alías, Angel FerRandez and Pascual LuCAS

Lie algebras of type $D_{4}$ over number fields

209

BRUCE ALLISON

Subsemigroups of completely simple semigroups

251

Anne Antonippillai and Francis PAStiJn

Studying links via closed braids. VI. A nonfiniteness theorem

JOAN Birman and William W. MENASCO

Minimal orbits at infinity in homogeneous spaces of nonpositive curvature

MARÍA J. DRUETTA

Generalized horseshoe maps and inverse limits

SARAH ElizABETH Holte

Determinantal criteria for transversality of morphisms

DAN LAKSOV and ROBERT SPEISER

Four dodecahedral spaces

PETER LORIMER

Semifree actions on spheres

MONICA NiCOLAU

Conformal deformations preserving the Gauss map

ENALDO VERGASTA

Hecke eigenforms and representation numbers of arbitrary rank lattices 371

LYNNE WALLING 